\title{
Data Rate and Throughput Analysis of Cooperative Cognitive Radio Under a Collision Model
}

\author{
Seyed Hossein Seyedmehdi and Ben Liang \\ Department of Electrical and Computer Engineering \\ University of Toronto, Canada \\ Email: \{hossein, liang\}@ comm.utoronto.ca
}

\begin{abstract}
We consider a cooperative cognitive radio system, where a secondary user may relay data for the primary user. Under the half-duplex assumption and packet collision model, the primary and secondary instantaneous data rates are derived, as a function of the power allocation factor by the secondary user to relay the primary user's data. We study the optimal power allocation to maximize the secondary user's instantaneous data rate and long-term throughput, without degrading the primary user's spectrum utilization. Our numerical results suggest that these two performance criteria entail drastically different optimal behaviors by the secondary user. Furthermore, we observe that employing successive interference cancellation in relaying can significantly increase the instantaneous data rate, but after optimizing the power allocation factor, the simple decode-andforward strategy almost always achieves the same throughput as the more complicated strategies.
\end{abstract}

\section{INTRODUCTION}

The performance of the cooperative cognitive radio (CCR) has been widely studied from an information theoretic perspective [1]-[3]. In a pioneering work, Devroye et al. [1] studied the data rate of the non-causal CR assuming a genie informs the secondary transmitter (ST) about the message of the primary transmitter (PT) prior to transmission. The causal CR model was later developed in [4], where the authors adopted a cooperative strategy for the $\mathrm{CR}$ and provided an inner bound on the joint achievable rates of the primary user (PU) and the secondary user (SU). In this model, the SU acts as a relay with a private message. The largest inner bound to-date for this model is derived in [5], but it involves many auxiliary random variables, thus making the rates intractable.

Most previous CCR performance studies are based on either impractical assumptions or models that are hard to implement. In an idealistic CCR, the ST can operate in full-duplex mode [2]. However, the full-duplex transceivers are impractical with today's technology. The inner bounds on the half-duplex CR was recently investigated in [6]. However, like other works on fundamental limits of the cognitive radio, this work also provides an intractably high-dimensional rate region that is too prohibitive for practical design purposes. Another implicit assumption in the aforementioned works is that two colliding messages at the receiver can be decoded as if they are perfectly synchronized. Holding this assumption is very cumbersome in reality: Decoding colliding packets are possible, but it requires highly sophisticated receivers and transmitters [7]. For instance, in [8], a cross-layer protocol called DAC is developed to circumvent the synchronization requirement which involves a complicated signal processing.

In this work, we consider a CCR model in which the SU can cooperate with the PU in half-duplex mode. Furthermore, neither primary receiver (PR), nor the secondary receiver (SR), can decipher two colliding packets. Hence, only one transmitter is allowed to transmit (interleaved transmission) to avoid collision at the receivers. Our physical channel model resembles that in [9]. The goal for the SU is to maintain the quality of service (QoS) of the PU, while optimizing its own spectrum utility (data rate and throughput). In this paper, we consider instantaneous data rate and long-term throughput as performance metrics. An interleaved transmission is adopted to avoid packet collision.

To best of our knowledge, this work is the first to investigate such a problem. Nevertheless, maximizing the performance of the SU while maintaining that of the PU has been previously studied in various literatures. For example, [10] proposes a distributed power/channel allocation algorithm to maximize the down link coverage and throughput while maintaining the interference to the PU below a certain threshold. In this work, the ST does not cooperate in transmitting the PU data. In [11], adaptive user cooperation in heterogeneous cognitive relay system is considered assuming that colliding packet can always be decoded. In [12], a CCR model is investigated in which there are one single PT and multiple STs. In the adopted cooperative scheme, the time is divided into three unequal partitions. In the first time slot, the PT broadcasts its message to STs only, and all STs are required to decode it. In the second time slot, the PT and all STs transmit this message simultaneously to the PR. In the third time slot, the channel is relinquished to the SUs and each SU utilizes it based on a pricing scheme. Unlike our model, the colliding packets are assumed to be decoded, and only DF without SIC is considered.

The main contributions of this work can be summarized as follows:

- Decode-and-forward (DF) and amplify-and-forward (AF) strategies for the CCR are investigated as two prevailing strategies. Different decoders with successive interference cancellation (SIC) and without SIC are considered. For each cooperative strategy, analytical closed forms for the optimal power allocation and maximum achievable rate for the SU is obtained.

- The throughput of the system is investigated, and through 
simulation it is shown that in most cases, with optimal power allocation between the superpositioned PT and ST data, the non-sophisticated DF method performs as well as sophisticated DF methods with SIC.

\section{System Model and Problem Statement}

We consider a CCR system as depicted in Fig. 1. In this model, there is a PU with one transmitter and one receiver. The PT has a message to transmit, and the PR receives and decodes that message. There is also a SU with one transmitter, ST, and one receiver, SR. For convenient indexing, PT, ST, PR, SR are denoted by nodes $1, \cdots, 4$. When the ST cooperates, then the time is divided into alternating slots. In odd slots, the PT transmits its own signal whereas in even slots, the ST transmits a superimposed signal containing its own message and a version of what it received from the PT in the previous slot.

To mathematically model this communication channel, let $\tilde{X}_{i}$ indicate the transmitted signal by node $i$, and $\tilde{Y}_{i}$ indicate the received signal by node $i, i=1,2,3,4$. Also, let $\tilde{h}_{i j}$ be the channel gain between nodes $i$ and $j$. The relationship between transmitted signals and received signals can be expressed as

$$
\left[\begin{array}{c}
\tilde{Y}_{2} \\
\tilde{Y}_{3} \\
\tilde{Y}_{4}
\end{array}\right]=\left[\begin{array}{ll}
\tilde{h}_{21} & 0 \\
\tilde{h}_{31} & \tilde{h}_{32} \\
\tilde{h}_{41} & \tilde{h}_{42}
\end{array}\right]\left[\begin{array}{c}
\tilde{X}_{1} \\
\tilde{X}_{2}
\end{array}\right]+\left[\begin{array}{c}
\tilde{Z}_{2} \\
\tilde{Z}_{3} \\
\tilde{Z}_{4}
\end{array}\right]
$$

where $\tilde{Z}_{i}$ is an additive white Gaussian noise at node $i$ with power $E\left[\tilde{Z}_{i}^{2}\right]=\tilde{N}_{i}$. The channel gains $\tilde{h}_{i j}$ are assumed to be real; the extension to complex channel gains is straight forward and is ignored here. The transmitters are subjected to the power constraint $E\left[\tilde{X}_{i}^{2}\right] \leq \tilde{P}_{i}$. The Gaussian noise requires the transmitted signals to be Gaussian as well since the normal distribution maximizes the entropy for a given variance [13, Theorem 8.6.5]. We term this channel as a Gaussian interference channel hereafter. In this model, it is assumed that the PT can communicate with the PR with the Shannon rate which in our model is equal to $0.5 \log (1+S N I R)$ bits per channel use where the logarithms are taken in base 2, and the SNIR is the ratio of the received signal power at the PR over the total noise and interference power, i.e., $S N I R=\tilde{h}_{31}^{2} \tilde{P}_{1} /\left(\tilde{h}_{32}^{2} \tilde{P}_{2}+\tilde{N}_{1}\right)$.

To make the analysis more tractable, we now present the notion of the standard channel. It has been shown (see [14] for example) that by applying a scaling transformation on the parameters and input signals, the resulting changes in output signals can be compensated by constant scaling factors. That is, two Gaussian interference channels related by this scaling transformation are equivalent in terms of performance metrics. Let (1) represent the original channel input output relationship. The channel characterized by the following input output relationship has the same performance metrics as that in (1).

$$
\left[\begin{array}{l}
Y_{2} \\
Y_{3} \\
Y_{4}
\end{array}\right]=\left[\begin{array}{ll}
a & 0 \\
1 & c \\
b & 1
\end{array}\right]\left[\begin{array}{l}
X_{1} \\
X_{2}
\end{array}\right]+\left[\begin{array}{l}
Z_{2} \\
Z_{3} \\
Z_{4}
\end{array}\right],
$$

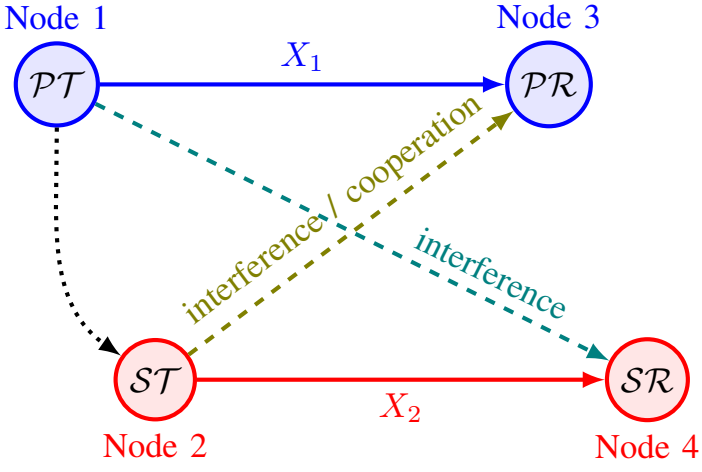

Fig. 1. Mnemonic channel model for the cooperative cognitive radio. In this figure, $\mathcal{P} \mathcal{T}, \mathcal{S} \mathcal{T}, \mathcal{P} \mathcal{R}, \mathcal{S R}$ represent the primary transmitter (PT), secondary transmitter (ST), primary receiver (PR), and secondary receiver (SR) respectively.

where

$$
a=\frac{\tilde{h}_{21} \sqrt{\tilde{N}_{3}}}{\sqrt{\tilde{N}_{2}}}, b=\frac{\tilde{h}_{41} \sqrt{\tilde{N}_{3}}}{\tilde{h}_{31} \sqrt{\tilde{N}_{4}}}, c=\frac{\tilde{h}_{32} \sqrt{\tilde{N}_{4}}}{\tilde{h}_{42} \sqrt{\tilde{N}_{3}}},
$$

and the new transmitted signals and powers are

$$
X_{1}=\frac{\tilde{h}_{31} \tilde{X}_{1}}{\sqrt{\tilde{N}_{3}}}, X_{2}=\frac{\tilde{h}_{42} \tilde{X}_{2}}{\sqrt{\tilde{N}_{4}}}, P_{1}=\frac{\tilde{h}_{31}^{2} \tilde{P}_{1}}{\tilde{N}_{3}}, P_{2}=\frac{\tilde{h}_{42}^{2} \tilde{P}_{2}}{\tilde{N}_{4}},
$$

and the noise powers are normalized to one. For convenience, we will analyze the standard channel hereafter; nonetheless, the results are easily transformable to the form that contains the original channel's parameters.

Let the $R_{1}$ indicate the information theoretic rate for the PU. Similarly, let $R_{2}$ be the SU's rate by which the ST conveys the information to the SR. As mentioned before, the PU has the right to use the spectrum, and its rate may not be degraded by the interference incurred by the SU. The data rate of the PU when it fully utilizes the channel is

$$
R_{1}^{\star}=\frac{1}{2} \log \left(1+P_{1}\right) .
$$

If the ST transmits concurrently with power $P_{2}$, the rate of the PU diminishes to $R_{1}=0.5 \log \left(1+P_{1} /\left(1+c^{2} P_{2}\right)\right)$.

The SU is, however, a smart cognitive user that can cooperate with the PU. It can play a twofold role: On the one hand, if the ST selflessly allocates all of its power to relay the message of the PT, it can enhance the performance of the PU while achieving zero rate for the ST; on the other hand, if the ST selfishly allocates all of its power to transmit its message, the rate of the PU decreases.

\section{Achievable Rates of THe CCR}

In this section, we investigate the achievable data rates of the CCR modeled in Section II under DF and AF schemes.

\section{A. Decode-and-Forward Strategies}

In the absence of the SU, let $2 n$ be the number of channel uses for a packet of data conveying $\bar{b}$ bits of uncoded information. But when the SU cooperates, the PU only transmits half of the time, i.e., in $n$ channel uses, and the SU transmits in 
the second half of the time. Each half time is called one time slot. It is noteworthy that $n$ is assumed to be large enough such that the Shannon capacity results can be applied here. In more precise words, in first time slot, PT transmits

$$
X_{1}^{n}=\left(X_{1,1}, X_{1,2}, \cdots, X_{1, n}\right)
$$

where $X_{1}^{n}$ denotes the PT's message (packet) vector with length $n$, and $X_{1, i}$ represents the message of the PT at time instance $i$. In the second time slot, the ST transmits

$$
X_{2}^{n}=\left(X_{2, n+1}, X_{2, n+2}, \cdots, X_{2,2 n}\right) .
$$

When it is clear from the context we drop the time index $i$ for $X_{1, i}$ or $X_{2, i}$. For a given time instance $i \in\{1, \cdots, n\}$, let $X_{1}^{\prime}$ be an i.i.d. normal random variable with mean zero and unit variance. The output of the PT can be represented as

$$
X_{1}=\sqrt{P_{1}} X_{1}^{\prime}
$$

In the decode and forward (DF) scheme, the ST decodes the message of the PT transmitted in the first time slot, i.e., $X_{1}^{n}$. In the second time slot, at time index $j=i+n$, the transmitted symbol by the ST can be expressed as

$$
X_{2}=\sqrt{\beta P_{2}} X_{1}^{\prime}+\sqrt{\bar{\beta} P_{2}} X_{2}^{\prime}
$$

where $X_{2}^{\prime}$ is a Gaussian i.i.d. random variable with mean zero and unit variance and $\bar{\beta}=1-\beta$. As can be seen, the ST's power is split into two parts: first part, $\beta P_{2}$, is used to forward the message of PT; the second part, $\bar{\beta} P_{2}$, is used to transmit the message of the ST. The power allocation factor $\beta$ plays a rate tuning role: intuitively, increasing $\beta$ (allocating more power to the PT's message and less power to the ST's message) results in increasing $R_{1}$ and decreasing $R_{2}$.

Let $Y_{k}^{(1)}$ be the received signal at node $k$ in the first time slot at an arbitrary time index $i$, and $Y_{k}^{(2)}$ be the received signal at node $k$ in the second time slot at the respective time index $i+n$. Therefore, the received signals at the PR and the SR can be expressed as

$$
\begin{gathered}
Y_{3}=\left[\begin{array}{l}
Y_{3}^{(1)} \\
Y_{3}^{(2)}
\end{array}\right]=\left[\begin{array}{c}
\sqrt{P_{1}} \\
c \sqrt{\beta P_{2}}
\end{array}\right] X_{1}^{\prime}+\left[\begin{array}{c}
0 \\
c \sqrt{\bar{\beta} P_{2}}
\end{array}\right] X_{2}^{\prime}+\left[\begin{array}{c}
Z_{3}^{(1)} \\
Z_{3}^{(2)}
\end{array}\right], \\
Y_{4}=\left[\begin{array}{l}
Y_{4}^{(1)} \\
Y_{4}^{(2)}
\end{array}\right]=\left[\begin{array}{l}
b \sqrt{P_{1}} \\
\sqrt{\beta P_{2}}
\end{array}\right] X_{1}^{\prime}+\left[\begin{array}{c}
0 \\
\sqrt{\bar{\beta} P_{2}}
\end{array}\right] X_{2}^{\prime}+\left[\begin{array}{l}
Z_{4}^{(1)} \\
Z_{4}^{(2)}
\end{array}\right]
\end{gathered}
$$

where $Z_{k}^{(i)}$ is the white Gaussian noise at node $k$ in time slot $i$ with unit variance.

Since we impose the condition that the message of the PT must be decoded at the ST, the transmission rate of the PT should be less than the point-to-point capacity between the PT and the ST. This capacity can be written as $\frac{1}{2} C\left(a^{2} P_{1}\right)$ where the $\frac{1}{2}$ factor is due to the fact that the PT transmits half of the time, and $C(x)=\frac{1}{2} \log (1+x)$. In addition, under this cooperative scheme, we implicitly assume that the rate of the PT is as described in (3); the following constraint must be satisfied:

$$
\frac{1}{2} \times C\left(a^{2} P_{1}\right) \geq R_{1}^{\star} \Rightarrow a^{2} \geq 2+P_{1} .
$$

In the rest of this chapter we investigate three methods of decoding: first, decoding without interference cancellation; second, decoding with SIC at the PR; third, decoding with SIC at the SR. Also, let the rate $R_{1}$ be associated to the number of bits per channel use that $X_{1}^{\prime n}$ conveys, and similarly, the rate $R_{2}$ be associated to that of $X_{2}^{\prime n}$.

1) Decode-and-Forward with no SIC: In DF method with no SIC, each receiver only decodes its intended message while treating the message of the other transmitter as interference. The following theorem characterizes an achievable rate region under this scheme.

Theorem 1: In the channel described in Section II, a reliable communication is feasible if

$$
\begin{aligned}
R_{1} & \leq \frac{1}{4} \log \frac{1+P_{1}+c^{2} P_{2}+c^{2} \bar{\beta} P_{1} P_{2}}{1+c^{2} \bar{\beta} P_{2}}, \\
R_{2} & \leq \frac{1}{4} \log \left(1+\frac{\bar{\beta} P_{2}}{1+\beta P_{1}}\right)
\end{aligned}
$$

where $0 \leq \beta \leq 1$.

Proof: Consider (4) and (5): the PR forms a augmented codeword $Y_{3}^{n}$ which is a matrix with 2 rows and $n$ columns. The first row is the $n$-tuple received in the first time slot, and the second row is the $n$-tuple received in the second time slot. Note that symbol $i$ from the first time slot and the corresponding symbol $i+n$ from the second time slot are in the same column (column $i$ ), and $i=1, \cdots, n$. Also, note that the message of the PT is encoded in the $n$-tuple $X_{1}^{\prime n}$. In each channel use, the amount of information bits of $X_{1}^{\prime n}$ that can be reliably decoded from $Y_{3}^{n}$ (when $n$ is large) is upper bounded by the mutual information between $X_{1}^{\prime}$ and $Y_{3}$, i.e., $I\left(Y_{3} ; X_{1}^{\prime}\right)$ [15]. Here, there is a repetition: the same information bits are conveyed in the second time slot; therefore, the actual rate for the codeword $X_{1}^{\prime n}$ is half of this mutual information term. In other words, under this scheme, the PR can decode $X_{1}^{\prime n}$ if

$$
\begin{aligned}
R_{1} \leq & \frac{1}{2} I\left(Y_{3} ; X_{1}^{\prime}\right)=\frac{1}{2}\left(h\left(Y_{3}\right)-h\left(Y_{3} \mid X_{1}^{\prime}\right)\right) \\
= & \frac{1}{2} h\left(\left[\begin{array}{c}
\sqrt{P_{1}} X_{1}^{\prime}+Z_{3}^{(1)} \\
\sqrt{\beta P_{2}} X_{1}^{\prime}+c \sqrt{\bar{\beta} P_{2}} X_{2}^{\prime}+Z_{3}^{(2)}
\end{array}\right]\right) \\
& -\frac{1}{2} h\left(\left[\begin{array}{c}
Z_{3}^{(1)} \\
c \sqrt{\bar{\beta} P_{2}} X_{2}^{\prime}+Z_{3}^{(2)}
\end{array}\right]\right) \\
= & \left.\frac{1}{4} \log \left|\begin{array}{cc}
1+P_{1} & c \sqrt{\beta P_{1} P_{2}} \\
c \sqrt{\beta P_{1} P_{2}} & 1+c^{2} P_{2}
\end{array}\right|\right) \\
& -\frac{1}{4} \log \left|\begin{array}{cc}
1 & 0 \\
0 & 1+c^{2} \bar{\beta} P_{2}
\end{array}\right| \\
= & \frac{1}{4} \log \frac{1+P_{1}+c^{2} P_{2}+c^{2} \bar{\beta} P_{1} P_{2}}{1+c^{2} \bar{\beta} P_{2}}
\end{aligned}
$$

where $I(\cdot ; \cdot)$ represents the mutual information, and $h(\cdot)$ is the differential entropy (see [13] for definitions), and [13, Theorem 8.4.1] is used to compute (13).

Using a similar argument, let the SR form a augmented codeword $Y_{4}^{n}$. The SR can decode the message $X_{2}^{\prime n}$ if $R_{2} \leq$ $\frac{1}{2} I\left(Y_{4} ; X_{2}^{\prime}\right)$. This mutual information can be computed, and (12) will be obtained. 
2) Decode-and-Forward with SIC at the PR: In the second time slot, the PT is broadcasting two messages, $X_{1}^{\prime}$ and $X_{2}^{\prime}$, to the PR and the SR. When the cross channel gain $c$ is greater than one, the channel formed between the ST, the PR, and the SR is a degraded broadcast channel; that is the signal received by the SR is a degraded version of that received by the PR. In this case, let the term weaker receiver be used for the SR, and the term stronger receiver be used for the PR. Cover [16] and Gallager [17] showed that the capacity achieving strategy for the degraded broadcast channel is as follows: The transmitter superimposes the message of the weaker receiver onto the message of the stronger receiver. The stronger receiver first decodes the message of the weaker receiver, and then utilizes this knowledge to mitigate the interference when it decodes its own message. The weaker receiver only decodes its intended message. When the channel is Gaussian, the superposition coding is simply performed by linearly combining two codewords.

Having this guide line in mind, the following scheme utilizes the advantage of successive cancellation at the PR: let the PR first decode the message of the ST $\left(X_{2}^{\prime}\right)$ and then subtract it from the received signal $Y_{3}$. In other words, the PR decodes $X_{2}^{\prime}$ first and then $X_{1}^{\prime}$, and the SR decode only $X_{2}^{\prime}$.

Theorem 2: In the channel described in Section II, a reliable communication is possible if

$$
\begin{aligned}
R_{1} \leq & \frac{1}{4} \log \left(1+P_{1}+c^{2} \beta P_{2}\right), \\
R_{2} \leq & \min \left\{\frac{1}{4} \log \left(\frac{1+P_{2}}{1+\beta P_{2}}\right),\right. \\
& \left.\frac{1}{4} \log \left(\frac{1+P_{1}+c^{2} P_{2}+c^{2} \bar{\beta} P_{1} P_{2}}{1+P_{1}+c^{2} \beta P_{2}}\right)\right\}
\end{aligned}
$$

for $0 \leq \beta \leq 1$.

Proof: We imitate the proving technique in the Theorem 1: First, the decoders form augmented codewords $Y_{3}^{n}$ and $Y_{4}^{n}$. Then, the PR decodes the message of the SU reliably if $R_{2} \leq 0.5 I\left(Y_{3} ; X_{2}^{\prime}\right)$. Having decoded $X_{2}^{\prime n}$, the PR can utilize it as a known information to decode the message of the PU. The message of the PU can be decoded without error if $R_{1} \leq 0.5 I\left(Y_{3} ; X_{1}^{\prime} \mid X_{2}^{\prime}\right)$. Finally, the SR can decode the message $X_{2}^{\prime n}$ if $R_{2} \leq 0.5 I\left(Y_{4} ; X_{2}^{\prime}\right)$. Considering both constraints on $R_{2}$, clearly, $R_{2} \leq \min \left\{0.5 I\left(Y_{3} ; X_{2}^{\prime}\right), 0.5 I\left(Y_{4} ; X_{2}^{\prime}\right)\right\}$ must be satisfied.

3) Decode-and-Forward with SIC at the SR: The DF method with SIC at the SR is analogous to that with SIC at the PR (Section III-A2) except that the interference cancellation is performed at the SR instead of the PR. The following theorem states the rates achieved by this method.

Theorem 3: For the channel model described in Section II, a reliable communication is feasible if

$$
\begin{aligned}
R_{1} \leq & \min \left\{\frac{1}{4} \log \left(\frac{1+P_{1}+c^{2} P_{2}+c^{2} \bar{\beta} P_{1} P_{2}}{1+c^{2} \bar{\beta} P_{2}}\right),\right. \\
& \left.\frac{1}{4} \log \left(\frac{1+b^{2} P_{1}+P_{2}+b^{2} \bar{\beta} P_{1} P_{2}}{1+\bar{\beta} P_{2}}\right)\right\}, \\
R_{2} \leq & \frac{1}{4} \log \left(1+\bar{\beta} P_{2}\right)
\end{aligned}
$$

for $0 \leq \beta \leq 1$.

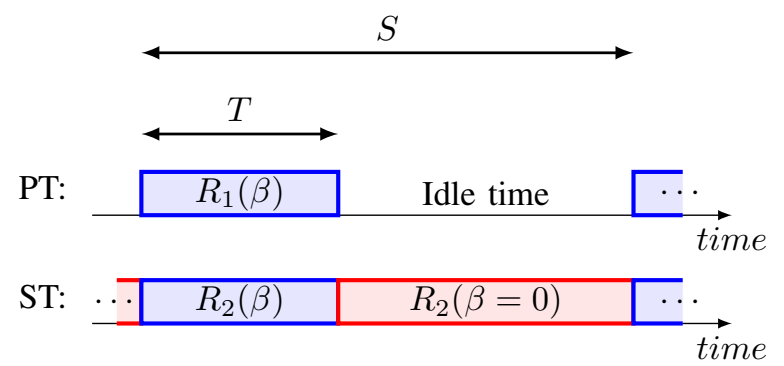

Fig. 2. Demonstration of the channel use over time by the PU and the SU. PT transmits only in $T / S$ portions of time, and the rest of the time the channel is idle for the SU's usage. $R_{1}$ and $R_{2}$ are the $\mathrm{PU}$ and the $\mathrm{SU}$ rates, and $\beta$ is the power allocation factor.

Proof: A similar proof technique to that of the Theorem 1 is used. The PR can decode $X_{1}^{\prime n}$ if $R_{1} \leq 0.5 I\left(Y_{3} ; X_{1}^{\prime}\right)$. The SR can decode $X_{1}^{\prime n}$ if $R_{1} \leq 0.5 I\left(Y_{4} ; X_{1}^{\prime}\right)$. Having the message of the PT known, the SR can decode the message from the ST if $R_{2} \leq 0.5 I\left(Y_{4} ; X_{2}^{\prime} \mid X_{1}^{\prime}\right)$.

\section{B. Amplify-and-Forward Strategies}

Similar to the DF scheme, in the AF scheme, the PT transmits its message (6) in the first time slot. The received signal by the $\mathrm{ST}$ in this time slot is $Y_{2}=Y_{2}^{(1)}=a \sqrt{P_{1}} X_{1}^{\prime}+Z_{2}^{(1)}$ where $Z_{2}^{(1)}$ is the white Gaussian noise with unit variance. In the second time slot at time index $j=i+n$, the ST transmits

$$
X_{2}=\sqrt{\beta P_{2}} \frac{Y_{2}}{\left\|Y_{2}\right\|}+\sqrt{\bar{\beta} P_{2}} X_{2}^{\prime}
$$

where $X_{2}^{\prime}$ is a Gaussian i.i.d. random variable with mean zero and unit variance, and $\left\|Y_{2}\right\|=\sqrt{E\left[\left|Y_{2}\right|^{2}\right]}$. Similar to the DF case, the power allocation factors $\beta$ in the interval $[0,1]$ is to be determined later.

In this paper we present only the results for AF with no SIC, $\mathrm{AF}$ with SIC at the PR, AF with SIC at the SR in Theorem 4, Theorem 5, and Theorem 6, respectively. The proof techniques are similar to those of theorems $1,2,3$, respectively, and they are omitted here.

Theorem 4: For the channel model described in Section II, a reliable communication is feasible if

$$
\begin{aligned}
R_{1} & \leq \frac{1}{4} \log \left(\frac{\left(1+c^{2} P_{2}\right)\left(\left(1+a^{2}+a^{2} P_{1}\right) P_{1}+1\right)-\beta a^{2} c^{2} P_{1}^{2} P_{2}}{1+a^{2} P_{1}+c^{2} P_{2}+a^{2} c^{2} P_{1} P_{2}-\beta a^{2} c^{2} P_{1} P_{2}}\right), \\
R_{2} & \leq \frac{1}{4} \log \left(\frac{1+P_{2}}{1+\beta P_{2}}\right)
\end{aligned}
$$

for $0 \leq \beta \leq 1$.

Theorem 5: A reliable communication in communication scenario modeled in Section II is feasible if

$$
\begin{aligned}
& R_{1} \leq \frac{1}{4} \log \left(\frac{1+P_{1}+a^{2} P_{1}+a^{2} P_{1}^{2}+\beta c^{2} P_{2}\left(1+P_{1}+a^{2} P_{1}\right)}{1+a^{2} P_{1}+\beta c^{2} P_{2}}\right), \\
& R_{2}=\min \{ \\
& \frac{1}{4} \log \left(\frac{1+\left(1+a^{2}\right)\left(P_{1}+c^{2} P_{1} P_{2}\right)+c^{2} P_{2}+P_{1}+a^{2} P_{1}^{2}+a^{2} c^{2} P_{1}^{2} P_{2} \bar{\beta}}{1+\left(1+a^{2}\right) P_{1}+a^{2} P_{1}^{2}+\beta c^{2} P_{2}\left(1+a^{2} P_{1}+P_{1}\right)}\right), \\
& \left.\frac{1}{4} \log \left(\frac{1+a^{2} P_{1}+P_{2}+a^{2} P_{1} P_{2}}{\left(1+a^{2} P_{1}\right)\left(1+\beta P_{2}\right)}\right)\right\}
\end{aligned}
$$

for $0 \leq \beta \leq 1$. 


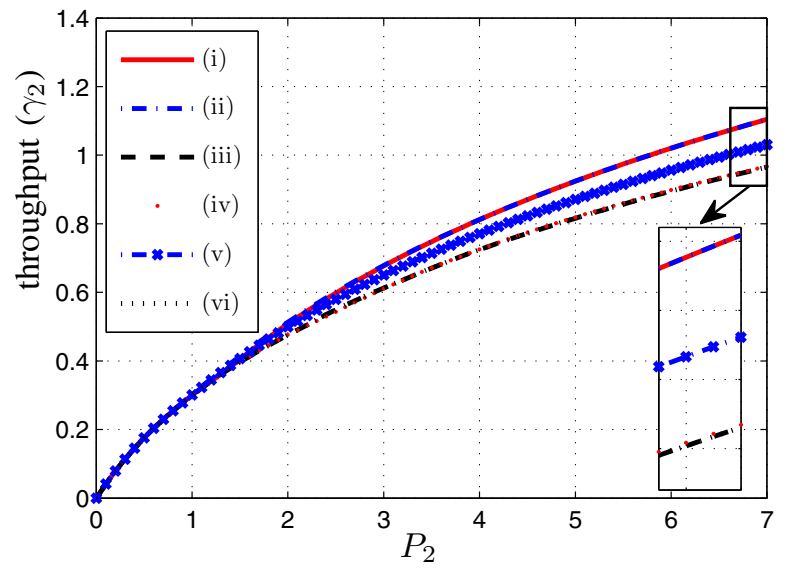

Fig. 3. (Legend corresponds to the strategies listed in Table I.) Throughput versus $P_{2}$ for six different strategies discussed in Sec. III-A and Sec. III-B. The channel parameters are as follows: $P_{1}=3 ; a=3 ; b=2 ; c=3$; the channel utilization factor $\zeta=0.4$. In this figure, (i) lies on top of (ii), and (iii) lies on top of (vi).

Theorem 6: In the communication channel described in Section II, an error-free communication is possible if

$$
\begin{aligned}
& R_{1} \leq \min \{ \\
& \frac{1}{4} \log \left(\frac{1+P_{1}\left(a^{2}+b^{2}\right)\left(1+P_{2}\right)+a^{2} b^{2} P_{1}^{2}+P_{2}+a^{2} b^{2} P_{1}^{2} P_{2} \bar{\beta}}{1+a^{2} P_{1}+P_{2}+a^{2} P_{1} P_{2} \beta}\right), \\
& \left.\frac{1}{4} \log \left(\frac{\left(1+c^{2} P_{2}\right)\left(\left(1+a^{2}+a^{2} P_{1}\right) P_{1}+1\right)-\beta a^{2} c^{2} P_{1}^{2} P_{2}}{1+a^{2} P_{1}+c^{2} P_{2}+a^{2} c^{2} P_{1} P_{2}-\beta a^{2} c^{2} P_{1} P_{2}}\right)\right\}, \\
& R_{2} \leq \frac{1}{4} \log \left(1+\frac{\bar{\beta} P_{2}\left(1+a^{2} P_{1}\right)}{1+a^{2} P_{1}+\beta P_{2}}\right)
\end{aligned}
$$

for $0 \leq \beta \leq 1$.

To conclude this section, we define a special power allocation factor $\beta_{\mathrm{R}}$ that maximizes the rate and compute it for different strategies presented in Theorems 1 to 6 .

Definition 1: A rate maximizing power allocation factor $\beta_{\mathrm{R}}$ is the power allocation factor $\beta$ adopted by the ST that maximizes the rate achieved by the $\mathrm{SU}, R_{2}$, while maintaining the interference-free rate for the PU as described in (3).

It can be verified that the rates $R_{1}$ obtained in Theorems 1 to 6 are monotonically increasing functions of $\beta$ whereas the ST's rate, $R_{2}$, obtained in those theorems are monotonically decreasing functions of $\beta$. Moreover, the data rate of the PT must at least be equal to that in (3), and the optimal power allocation can be obtained by comparing (3) and $R_{1}$ in each scheme. These optimal power allocations $\beta_{\mathrm{R}}$ are computed and listed in Table I. Note that these values for $\beta_{\mathrm{R}}$ are only valid if they are in the interval $[0,1]$; otherwise, the ST refrains from transmitting, and the PT utilizes both time slots.

\section{Throughput Analysis of the CCR}

In the previous section, we studied the data rate of the SU and showed how it varies with respect to different channel parameters when the $\mathrm{SU}$ is cooperating with the PU. An equally important performance metric for the wireless channels is the throughput, here defined as the time averaged number of uncoded bits transmitted over a relatively long period of time.

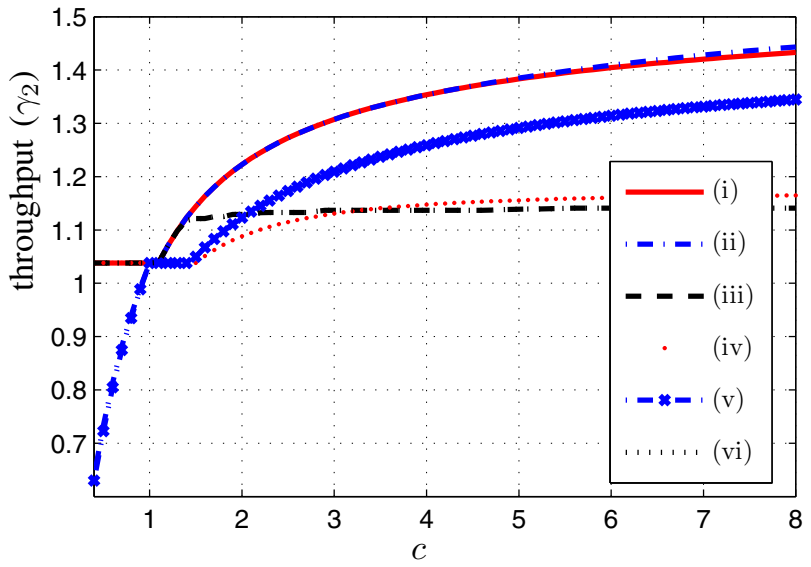

Fig. 4. (Legend corresponds to the strategies listed in Table I.) Throughput versus the channel gain $c$ for six different strategies discussed in Sec. III-A and Sec. III-B. The channel parameters are as follows: $P_{1}=3 ; P_{2}=10$; $a=3 ; b=2 ; c=3$; the channel utilization factor $\zeta=0.4$. In this figure, (iii) and (vi) lay on top of each other.

As illustrated in Fig. 2, let the time between two consecutive messages of the PU be $S$. We assume the required throughput of the PU is $\zeta S R_{1}^{\star}$ bits where $0<\zeta<1$ is the channel utilization factor (duty cycle), and $R_{1}^{\star}$ is the maximum point to point data rate obtained in (3), i.e., the PT can transmit the message to the PR in duration $\zeta S$. Hence, for the rest of the time, $(1-\zeta) S$, the channel is idle and can be utilized by other users.

Consider a slotted transmission as modeled in Section II. If the ST cooperates with the PT, the rate at which the PU can convey the data changes, and so does the time the PT requires to transmit $\zeta S R_{1}^{\star}$ bits. Let $R_{1}(\beta)$ be the rate achievable by the PU when the ST's power allocation factor is $\beta$ (see (7), (20)). Also, let $R_{2}(\beta)$ be the rate of the SU with power allocation factor $\beta$. Note that $R_{2}(0)$ is a special case meaning that the ST is either transmitting with full rate $0.5 \log \left(1+P_{2}\right)$ if the channel is ideal or not transmitting if the PT is transmitting.

Denote by $T$ the time duration that the PU requires within $S$, to achieve the same throughput $\zeta S R_{1}^{\star}$ with the rate $R_{1}(\beta)$. Then, the following constraint must hold to maintain the throughput of the PU intact:

$$
T R_{1}(\beta)=\zeta S R_{1}^{\star} .
$$

Depending on the chosen $\beta, T$ may be less or greater that $\zeta S$. In other words, the SU can adjust the PU's total transmission time. Note that since $T<S, R_{1}(\beta)$ must be greater than $\zeta R_{1}^{\star}$.

The overall throughput of the SU when it cooperates with the PU can be computed as follows:

$$
\begin{aligned}
\gamma_{2} & =\frac{1}{S}\left(T R_{2}(\beta)+(S-T) R_{2}(0)\right) \\
& =\frac{\zeta R_{1}^{\star}}{R_{1}(\beta)}\left(R_{2}(\beta)-R_{2}(0)\right)+R_{2}(0) .
\end{aligned}
$$

By substituting the $R_{1}(\beta)$ and $R_{2}(\beta)$ expressions from Theorems 1 to 6 into (27), a closed-form expression for the SU throughput can be obtained. 
TABLE I

LIST OF COOPERATIVE STRATEGIES STUDIED IN THIS PAPER

\begin{tabular}{|c|c|c|}
\hline Legend & Cooperative Strategy & Rate Maximizing Power Allocation Factor $\beta_{\mathrm{R}}$ \\
\hline $\begin{array}{l}\text { (i) } \\
\text { (ii) }\end{array}$ & $\begin{array}{c}\text { DF with no SIC } \\
\text { DF with SIC st the PR }\end{array}$ & $\begin{array}{c}\left(P_{1}+P_{1}^{2}+c^{2} P_{1} P_{2}+c^{2} P_{1}^{2} P_{2}\right) /\left(c^{2} P_{2}+c^{2} P_{1} P_{2}+c^{2} P_{1}^{2} P_{2}\right) \\
\left(P_{1}+P_{1}^{2}\right) /\left(c^{2} P_{2}\right)\end{array}$ \\
\hline (iii) & DF with SIC at the SR & $\min \left\{\frac{P_{1}\left(1+P_{1}\right)\left(1+c^{2} P_{2}\right)}{\left(1+P_{1}+P_{1}^{2}\right) c^{2} P_{2}}, \quad 1-\frac{2 P_{1}+P_{1}^{2}-P_{2}-b^{2} P_{1}}{b^{2} P_{1} P_{2}-P_{2}-2 P_{1} P_{2}-P_{1}^{2} P_{2}}\right\}$ \\
\hline (iv) & AF with no SIC & $\frac{1+\left(1+a^{2}\right)\left(P_{1}+c^{2} P_{1} P_{2}\right)+c^{2} P_{2}+a^{2} P_{1}^{2}+a^{2} c^{2} P_{1}^{2} P_{2}}{a^{2} c^{2} P_{2}\left(1+P_{1}+P_{1}^{2}\right)}$ \\
\hline (v) & AF with SIC at the PR & $\left(1+\left(1+a^{2}\right) P_{1}+a^{2} P_{1}^{2}\right) /\left(c^{2} P_{2}\left(a^{2}-1-P_{1}\right)\right)$ \\
\hline (vi) & AF with SIC at the SR & $\min \left\{\frac{\left(1+P_{2}\right)\left(\left(1+2 a^{2}+a^{2} P_{1}-a^{2} b^{2}\right) P_{1}+2-b^{2}\right)}{a^{2} P_{2}\left(1+2 P_{1}+P_{1}^{2}-b^{2} P_{1}\right)}, \quad \frac{1+\left(1+a^{2}\right)\left(P_{1}+c^{2} P_{1} P_{2}\right)+c^{2} P_{2}+a^{2} P_{1}^{2}+a^{2} c^{2} P_{1}^{2} P_{2}}{a^{2} c^{2} P_{2}\left(1+P_{1}+P_{1}^{2}\right)}\right\}$ \\
\hline
\end{tabular}

Definition 2: A throughput maximizing power allocation factor $\beta_{\mathrm{Tp}}$ is the power allocation factor $\beta$ that maximizes the throughput of the SU, $\gamma_{2}$ in (27), subject to maintaining the throughput of the PU as that in (26).

It is intractable to obtain analytical forms for $\beta_{\mathrm{Tp}}$, since $\gamma_{2}$ contains logarithms and polynomials. However, the availability of a closed-form formula for the SU's throughput allows us to compute $\beta_{\text {Tp }}$ by numerical solvers.

\section{NUMERICAL ILLUSTRATIONS}

In this section, we illustrate the effect of different channel parameters on the throughput of the CCR.

Fig. 3 shows the throughput of the SU for different values of $P_{2}$ for the cooperative schemes discussed in Secs. III-A and III-B. As shown, for all schemes, the throughput naturally increases as $P_{2}$ increases. It is interesting, however, that the relatively more complicated DF scheme with SIC at the PR has no advantage over the simple DF with no SIC, and both achieved the highest throughput. The second highest throughput is obtained by the AF with SIC at the PR scheme. Unlike for DF, some SIC helps in AF schemes. Note that the lowest performance is for AF with SIC at the SR together with DF with SIC at the SR, we conclude that SIC at the SR is not helpful.

Fig. 4 demonstrates the throughput of different strategies versus the crossover channel gain $c$ when $P_{2}$ is relatively high. We note that, when $c<1$, the cross channel between the ST and the PR is weaker than the forward channel between the ST and the SR, and the requirement of decoding the message of the SU at the PR is a bottleneck for the rate of the SU, thus reducing the rate and the throughput of the SU. Again, we observe that medium and large channel gain $c$ (e.g., $1<c<6$ in this figure), the DF with no SIC and the DF with SIC at the PR both achieve the same throughput. Moreover, when $c<1$, DF with no SIC performs the same as DF with SIC at the SR in terms of throughput. Therefore, the simple DF has the highest performance in both of those regions of $c$.

\section{CONCLUding REMARKS}

In this paper we studied a four-node cooperative cognitive radio system, in which the $\mathrm{SU}$ is allowed to have nonzero rate only if the performance the PU is not degraded. We considered data rate and average throughput as performance criteria. Under the collision model, we obtained the optimal power allocation for the ST and formulated the conditions under which the SU is allowed to cooperate. We then analyzed the throughput of the SU provided that the throughput of the PU does not degrade. As a counter intuitive observation, we observed that a simple DF strategy with no SIC achieves almost the same throughput as the more complicated strategies with SIC. This phenomenon becomes more evident when the $\mathrm{SU}$ transmission power increases.

\section{REFERENCES}

[1] N. Devroye, P. Mitran, and V. Tarokh, "Achievable rates in cognitive radio channels," Information Theory, IEEE Transactions on, vol. 52, no. 5, pp. 1813-1827, May 2006.

[2] A. Goldsmith, S. Jafar, I. Maric, and S. Srinivasa, "Breaking spectrum gridlock with cognitive radios: An information theoretic perspective," Proceedings of the IEEE, vol. 97, no. 5, pp. 894 -914, may 2009.

[3] N. Devroye, M. Vu, and V. Tarokh, "Cognitive radio networks," Signal Processing Magazine, IEEE, vol. 25, no. 6, pp. 12 -23, november 2008.

[4] S. H. Seyedmehdi, Y. Xin, and Y. Lian, "An achievable rate region for the causal cognitive radio," in Proc. 45th Ann. Allerton Conf. on Communication, Control, and Computing, Monticello, IL, USA, Sep. 2007.

[5] S. Rini, D. Tuninetti, and N. Devroye, "New inner and outer bounds for the discrete memoryless cognitive interference channel and some capacity results," CoRR, vol. abs/1003.4328, 2010.

[6] D. Chatterjee, T. F. Wong, and O. Oyman, "On achievable rate regions for half-duplex causal cognitive radio channels," CoRR, vol. abs/1006.0964, 2010.

[7] J. Andrews, "Interference cancellation for cellular systems: a contemporary overview," Wireless Communications, IEEE, vol. 12, no. 2, pp. $19-29$, april 2005.

[8] X. Zhang and K. Shin, "Dac: Distributed asynchronous cooperation for wireless relay networks," in INFOCOM, 2010 Proceedings IEEE, 14-19 2010, pp. $1-9$.

[9] S. Hejazi and B. Liang, "Throughput analysis of multiple access relay channel under collision model," in INFOCOM, 2010 Proceedings IEEE, 14-19 2010, pp. $1-9$

[10] A. T. Hoang, Y.-C. Liang, and M. Islam, "Maximizing throughput of cognitive radio networks with limited primary users' cooperation," in Communications, 2007. ICC '07. IEEE International Conference on, 24-28 2007, pp. $5177-5182$.

[11] C. Sun and K. Ben Letaief, "User cooperation in heterogeneous cognitive radio networks with interference reduction," in Communications, 2008. ICC '08. IEEE International Conference on, 19-23 2008, pp. 3193 3197.

[12] J. Zhang and Q. Zhang, "Stackelberg game for utility-based cooperative cognitiveradio networks," in MobiHoc '09: Proceedings of the tenth ACM international symposium on Mobile ad hoc networking and computing. New York, NY, USA: ACM, 2009, pp. 23-32.

[13] T. M. Cover and J. A. Thomas, Elements of Information Theory, 2nd ed. Haboken, New Jersey: John Wiley \& Sons, 2006.

[14] A. Carleial, "Interference channels," Information Theory, IEEE Transactions on, vol. 24, no. 1, pp. 60 - 70, jan 1978.

[15] C. E. Shannon, "A mathematical theory of communicaion," Bell Sys. Tech. Journal, no. 27, pp. 379-423, 623-656, 1948.

[16] T. Cover, "Broadcast channels," Information Theory, IEEE Transactions on, vol. 18, no. 1, pp. 2-14, Jan. 1972.

[17] R. G. Gallager, "Capacity and coding for degraded broadcast channels," Probl. Peredachi Inf., vol. 10, no. 3, pp. 3-14, 1974. 